\title{
Analytical Study of Band Structure of Material Using Relativistic Concept
}

\author{
E. I. Ugwu ${ }^{1}$, M. I. Echi ${ }^{2}$ \\ ${ }^{1}$ Department of Industrial Physics, Ebonyi State University, Abakaliki, Nigeria \\ ${ }^{2}$ Department of Physics, University of Agriculture, Makurdi, Nigeria \\ Email: ugwuei@yahoo.com
}

Received March 21, 2013; revised April 21, 2013; accepted April 30, 2013

Copyright (C) 2013 E. I. Ugwu, M. I. Echi. This is an open access article distributed under the Creative Commons Attribution License, which permits unrestricted use, distribution, and reproduction in any medium, provided the original work is properly cited.

\begin{abstract}
In this paper, we present the study of band structure relativistically. Here, Dirac equation is formulated from Hamiltonian in which the formulation is found to contain a correction term known as spin-orbit coupling given as $\frac{\hbar}{2 m^{2} c^{2}}-\nabla V \cdot \nabla \psi+\frac{\hbar}{4 m^{2} c^{2}} \sigma \cdot(\nabla V x P \psi)$ that modifies the non-relativistic expression for the same formulation. This term leads to double spin-degeneracy within the first Brillioun zone which is a concept that is not found in other method of study of band structure of material.
\end{abstract}

Keywords: Bandstructure; Hamiltonian; Dirac Equation; Spin-Orbit Eigenfunction; Relativistic Concept; Spin-Degeneracy; Wavefunction; Correction-Term

\section{Introduction}

The study of band structure of materials non-relativistic concepts had a long time issue from the Bloch theorem of the form $\psi(r)=\mathrm{e}^{(\mathrm{i} k \cdot r)} U(r)$ applied to Schrodinger equation in which the obtained eigenstates and eigenvalues are periodic functions in $k$-space of which the information obtained from the reciprocal lattice gave the information on the band structure of the material [1-4].

In recent time, more work has been veraciously geared towards experimental study of band structure specially with regard to binary and ternary compound due to technological need of these materials with good band gap for use in solar cell and optoelectronics [4-6]. None of this approach coupled with other methods that had been on ground such as KKR, Pseudopontetial [6,7] etc. recognized spine-orbit.

However, it is important to mention here that in order to get a through picture of band structure of materials clearly, non-relativistic concept has to come in as it brings into consideration the spin-orbit splitting that was neglected in the relativistic study of band structure.

In this work, we present the analytical study of band structure of material using Dirac equation formulated from Hamiltonian in which case it will be found that the spin-orbit coupling term modifies the original non-relativistic Hamiltonian and reveals the fact that the classifi- cation of energy band by symmetry is completely altered as the spin-orbit term involving $2 \times 2$ Pauli's matrices is introduced and that it is no longer a question of phase shift of the wave function alone.

\section{Theoretical Frame Work: Band Theory}

Band structure as presented by KKR and APW and some methods depicted precisely the form of the secular equation in which Bloch wave function is expanded directly in plane wave. These two methods are related in a manner such that

$$
\operatorname{det}\left|\left\{(\kappa-K)^{2}-E\right\} \delta_{k K^{\prime}}+\Gamma_{k \Gamma^{\prime}}\right|
$$

has non-trivial solution in which the expression for Hamiltonian gives

$$
k_{m}\langle|H|\rangle k_{n}=\exp -\left[k+k_{m}\right]-\nabla^{2} V(r) \operatorname{expi}\left[k+k_{n}\right]
$$

with respect to matrix elements written in terms of plane wave where

$$
V(r)=\sum V_{k} \operatorname{expik} \cdot r
$$

In the case of KKR, emphasis is given on lattice structure and the scattering properties of a single Muffintin potential while APW centered on secular equation although both can be related when the phase shift analy- 
sis in which the scattering potential is of muffintin in nature.

In this case, a solution requiring incident plane wave, $\mathrm{e}^{\mathrm{i} k \cdot r}$ together with the scattered out going waves with the condition that $r V(r) \rightarrow 0$ as $r \rightarrow \infty$ is satisfied.

Under this condition, Green function method is used to obtain

$$
\Psi_{ \pm}=\frac{1}{2 \pi} \int \frac{\exp \pm \mathrm{i} k\left(r-r^{\prime}\right)}{\left|r-r^{\prime}\right|} V r^{\prime} \psi\left(r^{\prime}\right) \mathrm{d} r^{\prime}
$$

for which the required solution is

$$
\Psi=\exp i k z-\frac{m}{2 \pi \hbar^{2}} \int \exp -\mathrm{i} k \cdot r V\left(r^{\prime}\right) \mathrm{d} r^{\prime}
$$

That can be written in the form

$$
\Psi(r)=\mathrm{e}^{\mathrm{i} k \cdot z}+f(\Phi) \frac{\mathrm{e}^{\mathrm{i} k \cdot z}}{z}
$$

In the case of relativistic concept, we start with Hamiltonian form of Dirac equationwhich is written as

$$
\hbar \frac{\partial \psi}{\partial t}=H \Psi
$$

where $\psi$ expresses four component wave function

$$
\left(\rho(r)=\left(\begin{array}{l}
k_{1}(r) \\
k_{2}(r) \\
k_{3}(r) \\
k_{4}(r)
\end{array}\right)\right.
$$

And

$$
H=-c y \cdot P y_{\psi} m c^{2}+V(r)
$$

$P$ is momentum operator $\frac{\hbar \partial}{\mathrm{i} \partial r}$ while $V(r)$ is scalar potential. However, vector potential is neglected as it is considered to be zero [8]. $y_{\psi}$ is $4 \times 4$ matrix expressed simply in the form

$$
\left(\begin{array}{cccc}
1 & 0 & 0 & 0 \\
0 & 1 & 0 & 0 \\
0 & 0 & -1 & 0 \\
0 & 0 & 0 & -1
\end{array}\right) y_{j}=\left(\begin{array}{cc}
0 & \sigma_{j} \\
\sigma_{j} & 0
\end{array}\right)(j=1,2,3)
$$

$\sigma_{j}$ being Pauli's spin matrices

$$
\sigma_{1}=\left(\begin{array}{ll}
0 & 1 \\
1 & 0
\end{array}\right), \sigma_{2}=\left(\begin{array}{cc}
0 & -\mathrm{i} \\
\mathrm{i} & 0
\end{array}\right)
$$

With this, we write

$$
\Psi=\left(\begin{array}{l}
\phi \\
\psi
\end{array}\right)
$$

where $\phi$ and $\psi$ have components

$$
\phi=\left(\begin{array}{l}
x_{1} \\
x_{2}
\end{array}\right), \psi=\left(\begin{array}{l}
x_{3} \\
x_{4}
\end{array}\right)
$$

Then according to Schiff, we can write

$$
\mathrm{i} \hbar \frac{\partial \Psi}{\partial t}=\left[E+m c^{2}\right] \Psi
$$

We find that

$$
\left(E+m c^{2}-V\right) \phi+c \delta \cdot P \cdot \psi=0
$$

and

$$
[E-V] \psi+\subset \tau \cdot P \phi=0
$$

However, at low-energy limit, $\phi \ll \psi$ a situation where $\phi \sim(v / c) \psi$ where $v$ is the velocity of electron wavefunction as described by $\psi$. Based on the elimination of $\phi$ in favour of $\psi$ we have

$$
E \psi=\frac{1}{2 m}(\sigma \cdot P)\left[\mathrm{i}-\frac{E-V}{2 m c^{2}}\right]^{-1} \gamma \cdot P \psi+V \psi
$$

Using the relation

$$
\begin{gathered}
{\left[1+\frac{E-V}{2 m c^{2}}\right]^{-1}=1+\frac{E-V}{2 m c^{2}}} \\
P V=V p-\mathrm{i} \hbar \nabla V \\
(\sigma \cdot \nabla V)(\sigma \cdot P)=\nabla V \cdot P+\mathrm{i} \sigma(\nabla V \times P)
\end{gathered}
$$

This enables usto write Equation (17) as

$$
\begin{aligned}
E \psi= & {\left[1-\frac{E-V}{2 m c^{2}}\right] \frac{P^{2}}{2 m} \psi+V(r) \psi } \\
& -\frac{\hbar^{2}}{4 m^{2} c^{2}} \nabla V \cdot \nabla \psi+\frac{\hbar}{4 m^{2} c^{2}} \sigma(\nabla V \times P \psi)
\end{aligned}
$$

In this solution, we eliminate $E$ from the right hand side and observe that the last two terms are of the order $c^{-1}$ and

$$
\alpha=\frac{\mathrm{e}^{2}}{\hbar^{2}}=\frac{1}{137}
$$

Hence to order $\alpha^{2}$, we write $E-V=P^{2} / 2 m$ to obtain

$$
\begin{aligned}
E \psi= & \frac{P^{2}}{2 m} \psi-\frac{P^{2}}{8 m^{2} c^{2}}+V(r) \psi \\
& -\frac{\hbar^{2}}{4 m^{2} c^{2}} \nabla V \cdot \nabla \psi+\frac{\hbar}{4 m^{2} c^{2}} \sigma \cdot(\nabla V \times P \psi)
\end{aligned}
$$

Most interestingly all relativistic corrections as contained in Equation (17) are important when it has to do with heavy atoms and near the nucleus. In most case the correction from the second and fourth terms of the equation are omitted leaving just the simpler Hamiltonian. 


$$
H=\frac{P^{2}}{2 m}+V(r)+\frac{\hbar}{2 m^{2} c^{2}} \tau \cdot(\nabla V \times P)
$$

In this case it is found the only spin-orbit coupling term modifies the original non-relativistic Hamiltonian. This brings to the focus the idea of the fact that the classification of energy levels by symmetry is completely altered as the spin-orbit term involves $2 \times 2$ Pauli's matrices. Base on this case it is taken into account especially in forming the irreducible matrix representations that influence the symmetry group of the crystal.

From the study on the effect of relativistic corrections on energy levels in $\mathrm{PbT}_{e}$ by Pratt and Ferreira, there was no indicated evidence on modification of valence and conduction band extreme [9]. The only observed fact from their work which was experimental as compared to what is analytically observed isthat in the absence of spin-orbit coupling there is double spin-degeneracy at any point $k$ in the first Brillioun zone with the eigenfunction generalized as

$$
\left(\begin{array}{c}
u_{k}(r) \\
O
\end{array}\right) \mathrm{e}^{\mathrm{i} k v},\left(\begin{array}{c}
O \\
u_{k}(r)
\end{array}\right) \mathrm{e}^{\mathrm{i} k r}
$$

This degeneracy persists in the presence of spin-orbit coupling in as much as there exists crystal inversion centre in which case it is considered that $E(k)=E(-k)$. This explains the fact that the degeneracy is not altered by the presence of spin-orbit in accordance with $k \cdot p$ method when only first order perturbation theory is applied.

Since the second term on the right hand-side of Equation (21) denotes relativistic mass velocity correction, the fourth term has no classical analogue and it is then referred as the $e \cdot p$ term in which case e is referred to as effective field experienced by the electron. Though the two last term in Equation (21) do not introduce any further change in the scheme states are classified according to symmetry, they can cause important corrections to the band structure observed by Johnson, Conklin and Pratt in their study of $\mathrm{PbT}_{e}$ [10] from the foregoing analysis, Dirac equation for electrons in crystals is only solvable in approximate form of Equation (23) which is a reduced form of Equation (21).

It should be worth mentioning here that many of those methods of energy band theory as presented in the literature focused their study more in crystal potential not without considering the influence of the electron spinorbit as in Equation (23) rather more attention on influence of lattice structure and potential on the wave function as in Equations (3) and (5). Some of those methods such as APW, KKR and Pseudopotential also looked at the phase shift in function and quantum defect [11].

\section{REFERENCES}

[1] N. M. Aschroft and N. D. Mermin, "Solid State Physics Holt," Rinehart and Winston, 1976.

[2] P. M. Marcus, J. F. Janak and A. R. Williams, "Computational Methods in Band Theory," Plenum Press, New York, 1971. doi:10.1007/978-1-4684-1890-3

[3] B. Alder, S. Fernbach and M. Rotenburg, "Methods in Computational Physics," Energy Band in Solids, Vol. 8B Academic Press, New York, 1968.

[4] F. I. Ezema, A. B. C. Ekwealor and R. U. Osuji, Turkish Journal of Physics, Vol. 30, No. , 2006, pp. 157-163.

[5] T. Suziki, H. Kitazawa, M. Era, I. Ogoro, H. Shida, A. Yanase and T. Kasuya, "” Proceding of 4th International Conference on Crystal Field and Structural Effect in Electron System, 1981.

[6] T. Kasuya, In: T. Moriy, Ed., Electron Correlation and Magnetism in Narrow-Band Systems, Springer-Verlag Heidelberg; 1981, pp. 237-255. doi:10.1007/978-3-642-81639-0 24

[7] W. A. Harrison, "Pseudopotentials in the Theory of Metals Benjamin," New York, 1966.

[8] J. Zak, "The Irreducible Representations of Space Groups," New York, Amsterdam, 1969.

[9] G. W. Pratt and L. G. Ferreira, "Physics of Semiconductor," Dunod, Paris, 1964, p. 69.

[10] L. E. Jonson, J. B. Conklin and G. N. Pratt, Physical Review Letters, Vol. 11, No. 53, 1963.

[11] J. Williams and H. M. Norman, "Theoretical Solid State Physics,” Dover Publication, Inc., New York, 1974. 